03,11

\title{
Особенности фазовых переходов в висмутсодержащих упругонапряженных гетеросистемах AIGalnSbBi-InSb
}

\author{
(ㄷ Д.Л. Алфимова, Л.С. Лунин , М.Л. Лунина, А.С. Пащенко, О.С. Пащенко \\ Федеральный исследовательский центр Южный научный центр РАН, \\ Ростов-на-Дону, Россия \\ ฯ E-mail: lunin_Is@mail.ru
}

Поступила в Редакцию 28 марта 2019 г.

В окончательной редакции 28 марта 2019 г.

Принята к публикации 19 ноября 2019 г.

Обсуждаются особенности фазовых переходов в упругонапряженных гетеросистемах $\mathrm{Al}-\mathrm{Ga}-\mathrm{In}-\mathrm{Sb}-\mathrm{Bi}$ и подсистемах $\mathrm{In}-\mathrm{Sb}-\mathrm{Bi}$ и $\mathrm{Al}-\mathrm{In}-\mathrm{Bi}$. Исследованы изотермы для указанных гетеросистем и ее подсистем в интервале температур 653-923 К. Определены критические температуры и координаты критических точек спинодального распада твердого раствора AlGaInSbBi с учетом энергии когерентной деформации. Исследовано влияние упругих напряжений на термодинамическое поведение гетеросистемы $\mathrm{AlGaInSbBi}-\mathrm{InSb}$.

Ключевые слова: твердые растворы, фазовые переходы, изотермы солидуса, гетеросистемы, упругие напряжения, спинодальный распад.

DOI: 10.21883/FTT.2020.04.49114.440

\section{1. Введение}

Висмутсодержащие многокомпонентные твердые растворы на основе соединений $\mathrm{A}^{\mathrm{III}} \mathrm{B}^{\mathrm{V}}$ представляют особый интерес в силу многостороннего влияния висмута как на процесс кристаллизации материалов, так и на параметры приборов на их основе. Висмут, являясь полуметаллом, способствует уменьшению энергии запрещенной зоны легируемых им твердых растворов и смещает диапазон их фоточувствительности в инфракрасную область спектра [1], повышает морфологическую стабильность фронта кристаллизации и уменьшает отклонение твердых растворов от стехиометрии [2], и подобно кремнию и германию, в состоянии расплава имеет наибольшую плотность. Малая эффективная масса и аномально высокое значение средней длины свободного пробега электронов делают висмут чрезвычайно важным для изучения квантоворазмерных эффектов [3] и изготовления на их основе высокоскоростных, высокочувствительных оптоэлектронных приборов $[4,5]$.

Целью настоящей работы является анализ фазовых переходов в висмутсодержащих упругонапряженных гетеросистемах AlGaInSbBi-InSb и исследование влияния висмута на параметры термодинамической устойчивости к распаду твердых растворов $\mathrm{AlGaInSbBi,} \mathrm{выращенных}$ на подложках антимонида индия.

\section{2. Теоретический анализ}

Расчеты фазовых переходов для гетеросистемы $\mathrm{AlGaInSbBi}-\mathrm{InSb}$ и ее подсистем In-Sb-Bi и $\mathrm{Al}-\mathrm{In}-$ $\mathrm{Sb}-\mathrm{Bi}$ проводили в квазирегулярном приближении модели простых растворов [6]. Поверхность ликвидуса систем определялась методом визуально-термического анализа, заключающемся в непосредственном наблюдении растворения кристаллов в слитке шихты. Температура измеряется в момент исчезновения последней твердой частицы, принимаясь за ликвидусную.

Для моделирования фазовых превращений использовались следующие параметры:

1) значения параметров взаимодействия компонентов в жидкой фазе $\alpha_{A B}$ приведены в табл. 1;

2) значения параметров взаимодействия в квазибинарных твердых растворах $\alpha_{A B-A C}$ приведены в табл. 2;

3 ) значения температуры $T_{A B}$ и энтропии $\Delta S_{A B}$ плавления приведены в табл. 3 .

При формировании гетероструктуры происходит сопряжение фаз с различными кристаллическими решетками, т.е. когерентное сопряжение. Возникающие при когерентном сопряжении упругие напряжения смещают фазовые равновесия и существенно влияют на температуру фазовых переходов и характер кристаллизации, особенно на начальных стадиях жидкофазной эпитаксии. Следовательно, при расчетах фазовых переходов

Таблица 1. Параметры взаимодействия компонентов в жидкой фазе

\begin{tabular}{c|c}
\hline Система & $\alpha, \mathrm{J} / \mathrm{mol}$ \\
\hline $\mathrm{In}-\mathrm{Sb}$ & $-17225.00+3.44 \cdot T$ \\
$\mathrm{In}-\mathrm{Bi}$ & 1260.00 \\
$\mathrm{Ga}-\mathrm{Sb}$ & 685.00 \\
$\mathrm{Ga}-\mathrm{Bi}$ & 2950.00 \\
$\mathrm{Sb}-\mathrm{Bi}$ & 4441.40 \\
$\mathrm{In}-\mathrm{Ga}$ & 4452.00 \\
$\mathrm{In}-\mathrm{Al}$ & 4452.00 \\
$\mathrm{Ga}-\mathrm{Al}$ &
\end{tabular}


применялись уравнения когерентного равновесия для псевдобинарных и пятикомпонентных гетеросистем.

На рис. 1 приведены изотермы солидуса систем $\mathrm{Al}-\mathrm{In}-\mathrm{Sb}-\mathrm{Bi}(a)$ и $\mathrm{Al}-\mathrm{Ga}-\mathrm{In}-\mathrm{Sb}-\mathrm{Bi}(b)$ рассчитанные по уравнениям равновесной фазовой диаграммы (РФД) и по когерентной диаграмме состояния (КДС). Здесь приведены и экспериментальные данные. Видно, что учет упругих деформаций позволяет получить лучшее соответствие эксперименту. Следует отметить, что при малом содержании висмута в жидкой фазе $\left(x_{\mathrm{Bi}}^{l}<0.4 \mathrm{~mol}\right.$.frac.) расчеты по КДС отличаются от экспериментальных данных и хорошо сочетаются при большом содержании висмута ( $x_{\mathrm{Bi}}^{l}>0.4 \mathrm{~mol}$. frac.). По-видимому, при $x_{\mathrm{Bi}}^{l}<0.4$ развитие напряженных состояний слоя сдерживается частичной компенсацией деформаций, вызванных внедрением $\mathrm{Al}$ и $\mathrm{Bi}$.

$\mathrm{B}$ пятикомпонентной гетеросистеме $\mathrm{Al}-\mathrm{Ga}-\mathrm{In}-$ $\mathrm{Sb}-\mathrm{Bi}$ изопериод с $\mathrm{InSb}$ вообще отсутствует, и расчет по РФД, очевидно, малоинформативен. Расчет по КДС дает удовлетворительное согласие с экспериментом (рис. $1, b)$.

Таким образом, в большинстве случаев учет упругих напряжений на термодинамическое поведение системы позволяет осуществлять адекватное моделирование взаимодействия элементов в многокомпонентной гетеросистеме. Для практической реализации удобнее и нагляднее представлять фазовые переходы в многокомпонентных системах через концентрационные и температурные зависимости коэффициентов распределения компонентов.

На основе рассмотренной модели были проведены расчеты температурных и концентрационных зависимо-

Таблица 2. Параметры взаимодействия в квазибинарных твердых растворах

\begin{tabular}{c|c}
\hline Система & $\alpha, \mathrm{J} / \mathrm{mol}$ \\
\hline $\mathrm{InSb}-\mathrm{GaSb}$ & 9637 \\
$\mathrm{AlSb}-\mathrm{InSb}$ & 2520 \\
$\mathrm{AlSb}-\mathrm{GaSb}$ & 0 \\
$\mathrm{InBi}-\mathrm{GaBi}$ & 4360 \\
$\mathrm{InBi}-\mathrm{AlBi}$ & 3320 \\
$\mathrm{AlBi}-\mathrm{GaBi}$ & $5820-5900$ \\
$\mathrm{GaAs}-\mathrm{GaBi}$ & 1150 \\
$\mathrm{GaP}-\mathrm{GaBi}$ & 820
\end{tabular}

Таблица 3. Значения температуры и энтропии плавления бинарных компонентов твердых растворов

\begin{tabular}{c|r|c}
\hline Система & $T, \mathrm{~K}$ & $\Delta S, \mathrm{~J} /(\mathrm{mol} \cdot \mathrm{K})$ \\
\hline $\mathrm{InSb}$ & 798 & 59.95 \\
$\mathrm{AlSb}$ & 1338 & 61.91 \\
$\mathrm{GaSb}$ & 983 & 66.20 \\
$\mathrm{InBi}$ & 383 & 43.00
\end{tabular}
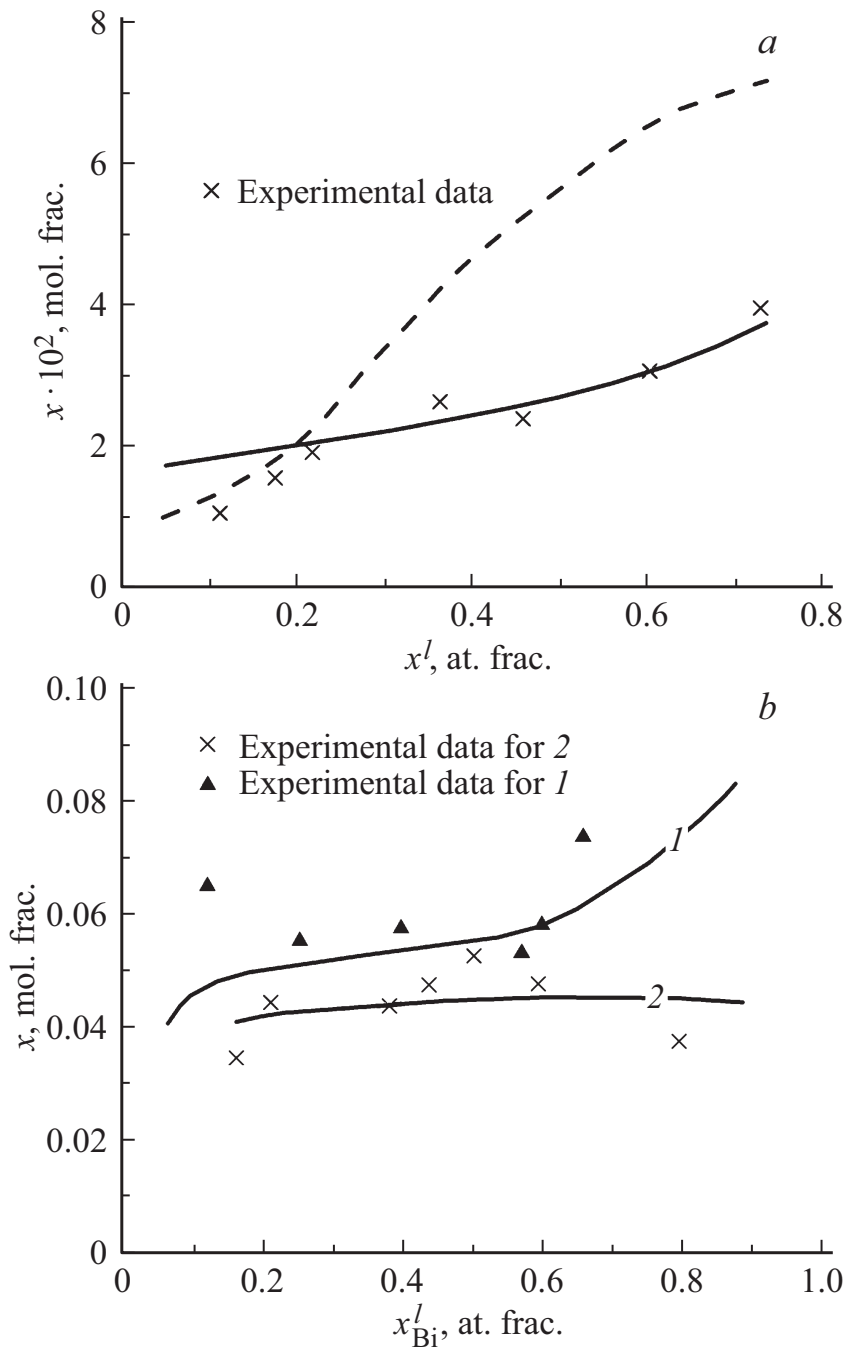

Рис. 1. $a-$ изотермы солидуса системы раствора Al-In-Sb-Bi при $T=653 \mathrm{~K}: 1-$ расчет по уравнениям РФД, 2 - расчет по уравнениям когерентной диаграммы состояния; $b$ - изотермы солидуса системы раствора $\mathrm{Al}_{x} \mathrm{Ga}_{y} \mathrm{In}_{1-x-y} \mathrm{Sb}_{1-z} \mathrm{Bi}_{z}$ при $T=715 \mathrm{~K}$. Содержание галлия $y<0.05 \mathrm{~mol}$. frac., висмута: $1-z=0.025 \mathrm{~mol}$. frac., $2-z=0.04$ mol. frac.

стей коэффициентов распределения компонентов, которые приведены на рис. 2-4.

Для терхкомпонентной системы $\operatorname{InSb}_{1-x} \mathrm{Bi}_{x} / \mathrm{InSb}$ на рис. 5 показаны зависимости $x_{i}^{l}$ от содержания висмута в твердом растворе (мольной доли $x$ ). Как видно из диаграмм, зависимости для индия и висмута симметричны относительно расплава $\operatorname{In}: \operatorname{Bi}(1: 1)$ и для них решения существуют в тем меньшей области х, чем выше температура. Таким образом, при температурах эпитаксии решения существуют только при $x \leq 0.1$ mol.frac.

На рис. 2 приведены зависимости коэффициентов распределения компонентов в ЧТР $\mathrm{Al}_{y} \mathrm{In}_{1-y} \mathrm{Sb}_{1-x} \mathrm{Bi}_{x}$. По сравнению с трехкомпонентными системами наличие в твердом растворе одновременно индия и алюминия приводит к снижению коэффициента распределения 
сурьмы, который падает с ростом мольных долей индия и висмута и слабо зависит от температуры. Небольшие значения коэффициентов распределения индия и очень малое значение коэффициента распределения висмута (рис. 2) делает возможным получение твердого раствора $\mathrm{Al}_{y} \mathrm{In}_{1-y} \mathrm{Sb}_{1-x} \mathrm{Bi}_{x} / \mathrm{InSb}$ при подпитке из обогащенного висмутом расплава.

Результаты расчета коэффициентов распределения компонентов в MTP $\mathrm{Al}_{y} \mathrm{Ga}_{z} \mathrm{In}_{1-y-z} \mathrm{Sb}_{1-x} \mathrm{Bi}_{x} / \mathrm{InSb}$ представлены на рис. 3. Общий характер зависимости тот же, однако интерес представляет возрастание коэффициента распределения сурьмы с увеличением доли алюминия в твердом растворе (рис. $3, b$ ). Для четырехвалентного твердого раствора (TP) $\mathrm{Al}_{y} \mathrm{In}_{1-y} \mathrm{Sb}_{1-x} \mathrm{Bi}_{x}$ наблюдается противоположная картина (рис. $2, b$ ), что свидетельствует о стабилизирующем влиянии галлия. Среднее значение $k_{\mathrm{Sb}}$ в обеих гетеросистемах примерно одинаково и равно 4 .
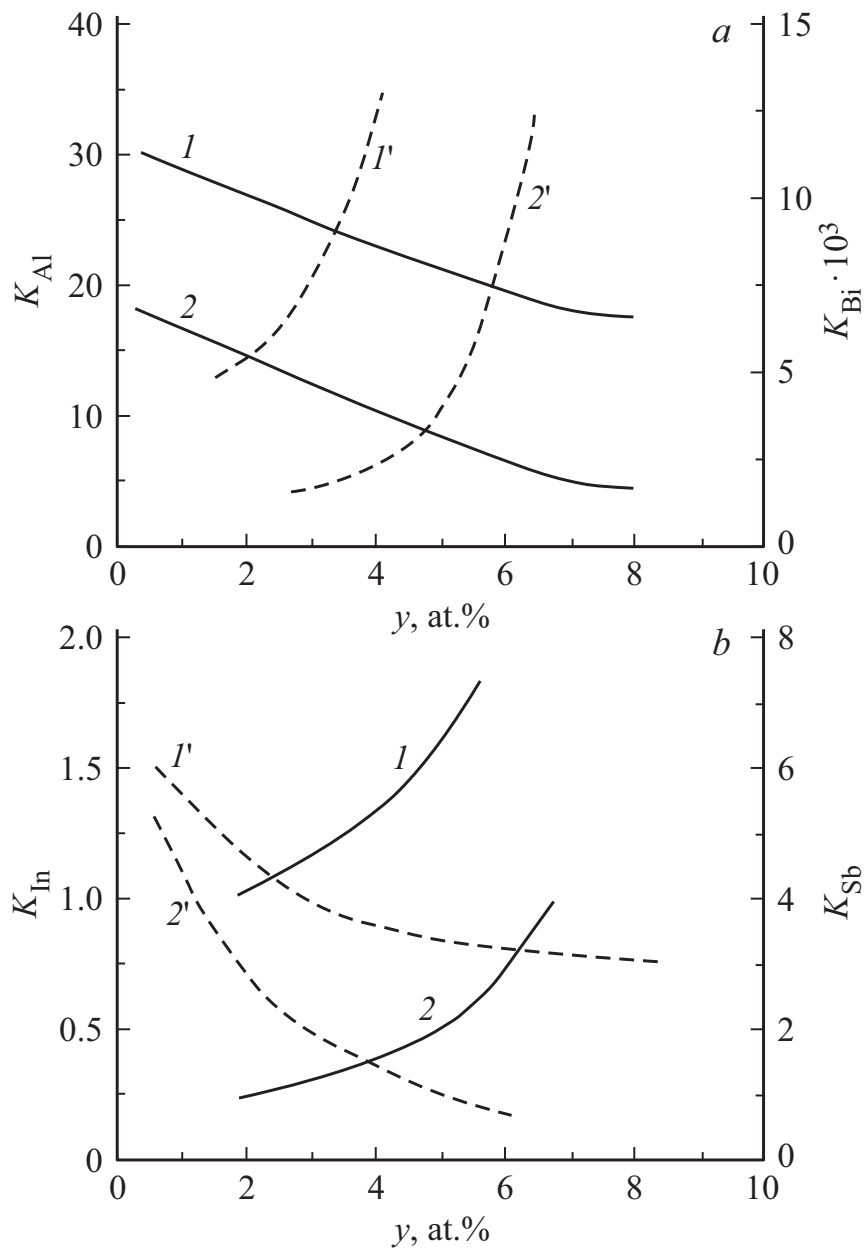

Рис. 2. $a-$ зависимость коэффициентов распределения $\mathrm{Al}$ (сплошные линии) и $\mathrm{Bi}$ (штриховые линии) в гетероструктуре $\mathrm{Al}_{y} \mathrm{In}_{1-y} \mathrm{Sb}_{1-x} \mathrm{Bi}_{x} / \mathrm{InSb}$ от состава $x$, at.\%: $1,1^{\prime}-1,2$; $2,2^{\prime}-2,5 ; b-$ зависимость коэффициентов распределения In (сплошные линии) и $\mathrm{Sb}$ (штриховые линии) в гетероструктуре $\mathrm{Al}_{y} \mathrm{In}_{1-y} \mathrm{Sb}_{1-x} \mathrm{Bi}_{x} / \mathrm{InSb}$ от состава $x$, at.\%: $1,1^{\prime}-1,2$; $22^{\prime}-2,5$.

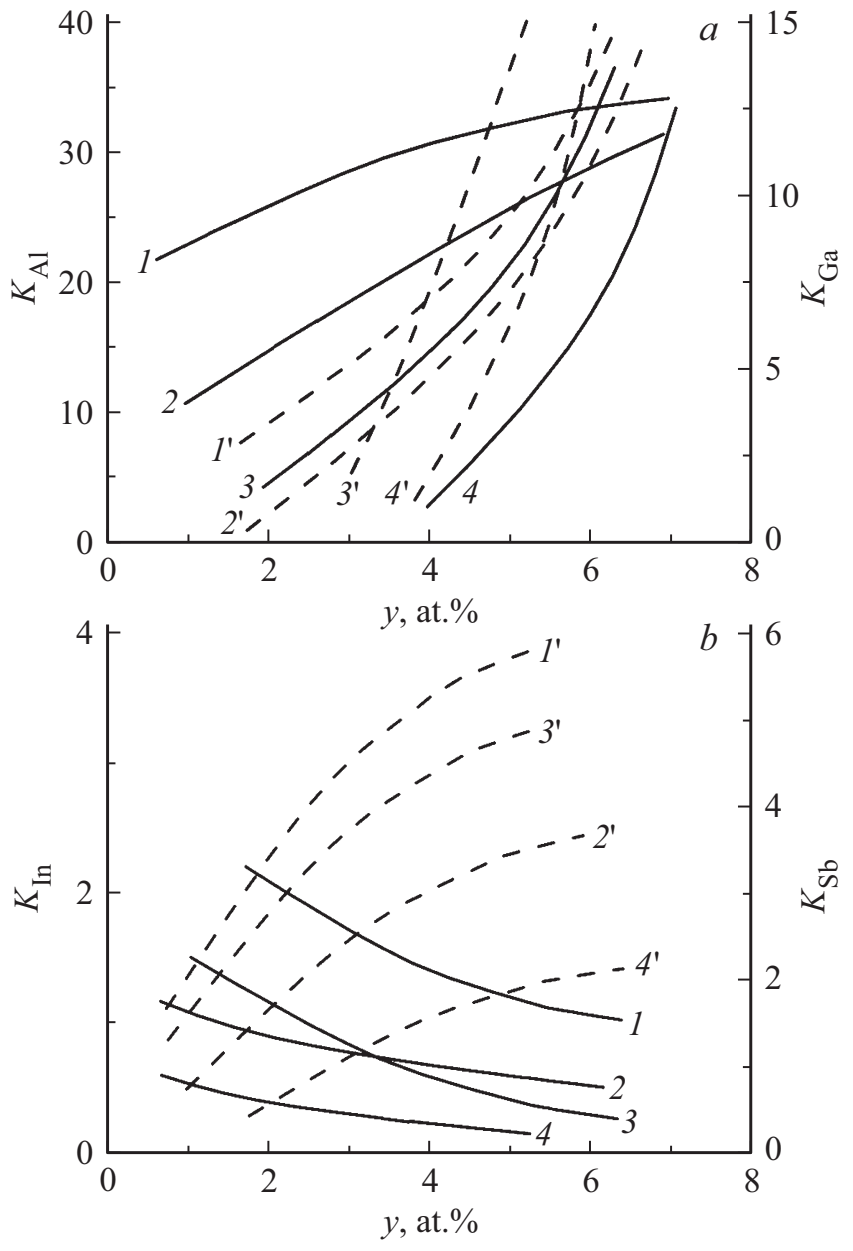

Рис. 3. $a-$ - зависимость коэффициентов распределения $\mathrm{Al}$ (сплошные линии) и $\mathrm{Ga}$ (штриховые линии) при $T=723 \mathrm{~K}$ в гетероструктуре $\mathrm{Al}_{y} \mathrm{Ga}_{1-y} \mathrm{In}_{1-y-z} \mathrm{Sb}_{1-x} \mathrm{Bi}_{x} / \mathrm{InSb}$ от состава, at.\%: $1,1^{\prime}-x=1,2, z=3,5 ; 2,2^{\prime}-x=2,5, z=3,5$; $3,3^{\prime}-x=1,2, z=8,2 ; 4,4^{\prime}-x=2,5, z=8,2 . b-$ зависимость коэффициентов распределения In (сплошные линии) и $\mathrm{Sb}$ (штриховые линии) при $T=723 \mathrm{~K}$ в гетероструктуpe $\mathrm{Al}_{y} \mathrm{Ga}_{1-y-z} \mathrm{In}_{1-y} \mathrm{Sb}_{1-x} \mathrm{Bi}_{x} / \mathrm{InSb}$ от состава, at.\%: 1, $1^{\prime}-$ $x=1,2, z=3,5 ; 2,2^{\prime}-x=2,5, z=3,5 ; 3,3^{\prime}-x=1,2$, $z=8,2 ; 4,4^{\prime}-x=2,5, z=8,2$.

Мольная доля висмута в твердом растворе может достигать 0.03 и уменьшается с ростом температуры. Для пятикомпонентного твердого раствоpa $\mathrm{Al}_{y} \mathrm{Ga}_{z} \mathrm{In}_{1-y-z} \mathrm{Sb}_{1-x} \mathrm{Bi}_{x} / \mathrm{InSb}$ особенностью является значительное отличие мольных долей до 0.30 (30\%) бинарных компонентов $\mathrm{GaBi}$ и $\mathrm{InBi}$, полученных в рамках квазихимического приближения парного взаимодействия, по сравнению с регулярным раствором. Растворимость висмута в пятикомпонентном ТР $\mathrm{Al}_{y} \mathrm{Ga}_{z} \mathrm{In}_{1-y-z} \mathrm{Sb}_{1-x} \mathrm{Bi}_{x} / \mathrm{InSb}$ заключена в узких пределах 0.01-0.03 mol.frac., при этом галлий воспроизводимо входит в состав твердого раствора до значений $z=0.082$. Поэтому фазовые диаграммы, приведенные на рис. 4, построены для мольных долей галлия 0.035 (кривые 1-3) и 0.082 (кривые 4-6). 


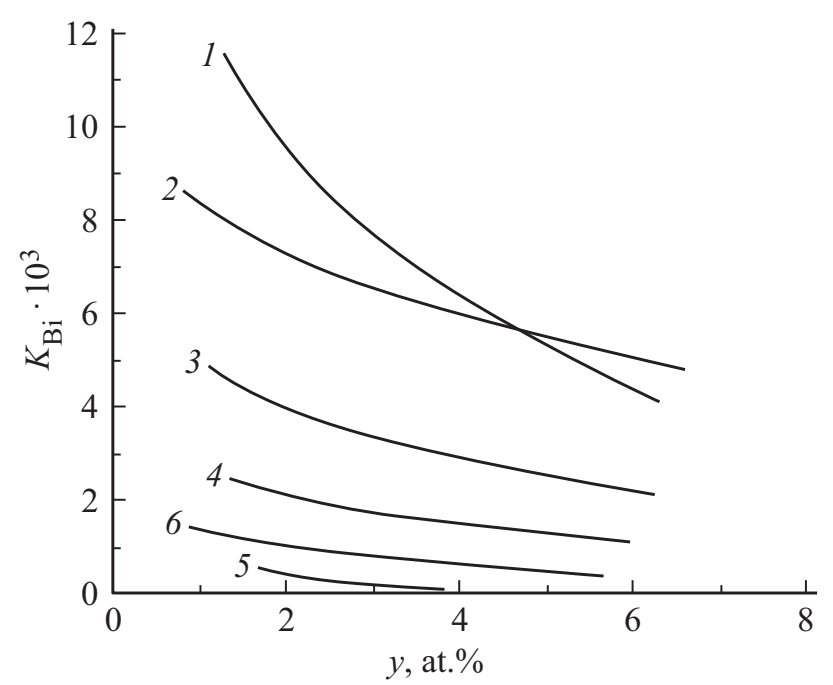

Рис. 4. Зависимость коэффициента распределения Ві при $T=723 \mathrm{~K}$ в гетероструктуре $\mathrm{Al}_{y} \mathrm{Ga}_{z} \mathrm{In}_{1-y-z} \mathrm{Sb}_{1-x} \mathrm{Bi}_{x} / \mathrm{InSb}$ от состава, at.\%: $1-x=1,2, z=3,5,2-x=1,8, z=3,5$, $3-x=2,5, z=3,5,4-x=1,2, z=8,2,5-x=1,8$, $z=8,2,6-x=2,5, z=8,2$.
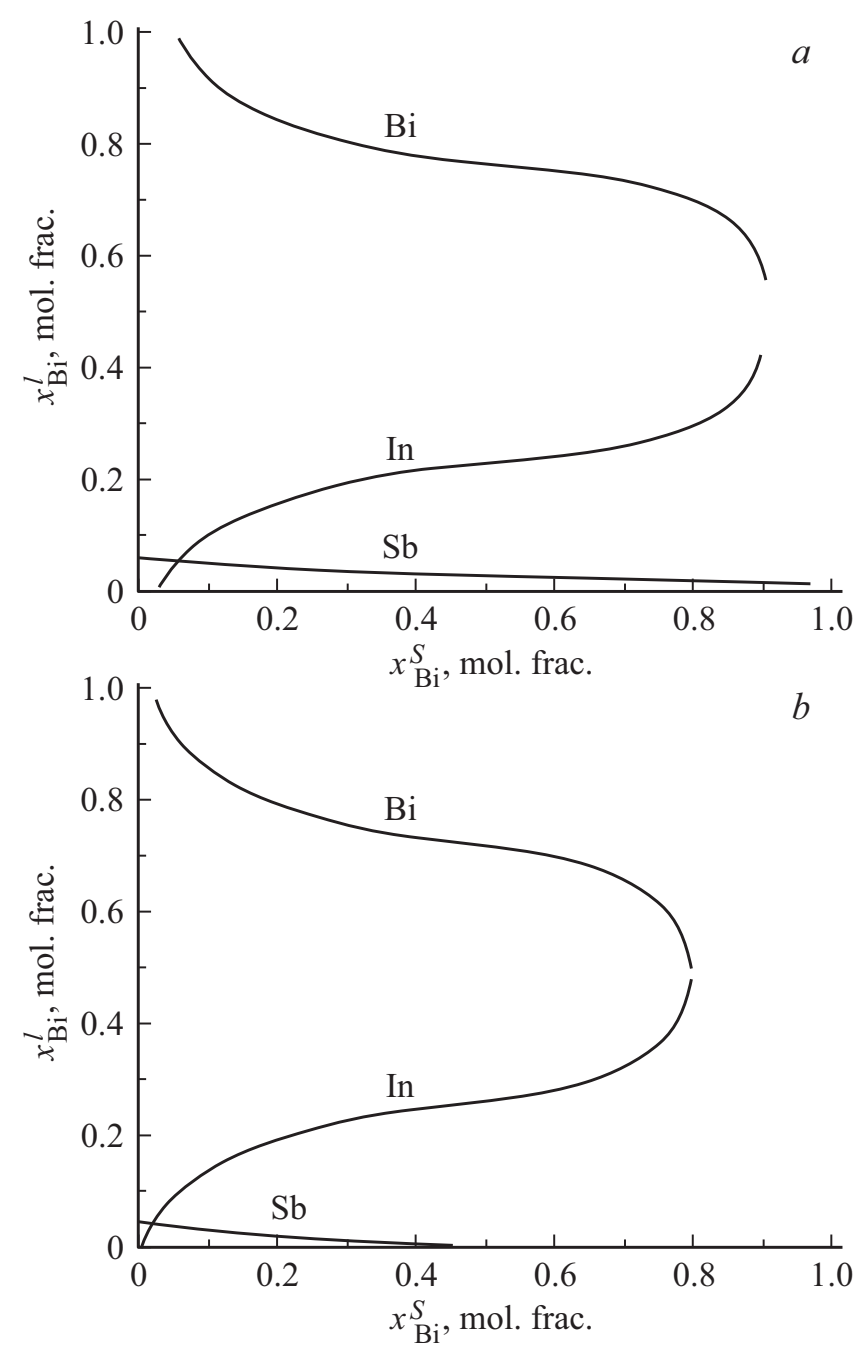

Рис. 5. Зависимость состава жидкой фазы твердого раствора $\operatorname{InBi}_{x} \mathrm{Sb}_{1-x}$ от мольной доли $x_{\mathrm{Bi}}: a-T=553 \mathrm{~K}$; $b-T=573 \mathrm{~K}$.
Зависимость состава жидкой фазы от температуры монотонна, поэтому детальная картина температурного характера приведена только для трехкомпонентного ТР InSbBi (рис. 5). Сравнивая зависимости, можно прийти к выводу, что повышение температуры не вызывает значительного изменения концентраций компонентов в жидкой фазе, а лишь приводит к сужению области, в которой уравнения, описывающие фазовые равновесия, имеют решения.

\section{3. Экспериментальная часть}

Выращивание твердых растворов AlInSbBi и $\mathrm{AlGaInSbBi} \mathrm{на} \mathrm{подложках} \mathrm{InSb} \mathrm{проводили} \mathrm{в} \mathrm{закрытой}$ системе в потоке водорода, очищенного в процессе диффузии, из жидкой фазы в поле температурного градиента (ЗПГТ) [6]. В качестве жидкой фазы использовали раствор-расплав сформированный из элементов In (ИН-000), Ga (ГЛ-000), Вi (ВИ-000), $\mathrm{Al}$ (АЛ-000) и бинарных соединений $\mathrm{InSb}$, $\mathrm{GaSb}$. Расчет навесок проводился по методике, описанной нами ранее [6], с учетом малярных масс элементарных компонентов. Все материалы, кроме галлия, подвергались химической обработке в смеси: $\mathrm{HNO}_{3}: \mathrm{H}_{2} \mathrm{O}(1: 1)$, промывке в дистиллированной воде и сушке в термостате при температурах $333-343 \mathrm{~K}$.

Твердые растворы AlGaInSbBi и AlInSbBi выращивали на монокристаллических пластинах антимонида индия с ориентацией по плоскостям (100) и (111) с концентрацией носителей $5 \cdot 10^{16} \leq n \leq 5 \cdot 10^{18} \mathrm{~cm}^{-3}$. Пластины, предварительно отшлифованные и полированные до 14-20 класса чистоты, подвергались химическому травлению $\mathrm{HCl}: \mathrm{HNO}_{3}: \mathrm{H}_{2} \mathrm{O}$ при температуре $343 \mathrm{~K}$ в течение $90 \mathrm{~s}$.

Толщина подложек после обработки составляла $350-400 \mu \mathrm{m}$. Далее пластины InSb промывали в дистиллированной воде и обрабатывали в парах толуола для удаления остатков органических веществ.

Выращивание твердых растворов $\mathrm{AlGaInSbBi}$ и AlInSbBi проводили при температурах $693 \leq T \leq 923 \mathrm{~K}$ и градиентах температуры $10 \leq G \leq 30 \mathrm{~K} / \mathrm{cm}$.

Экспериментальные исследования поверхности ликвидуса системы $\mathrm{Al}-\mathrm{Ga}-\mathrm{In}-\mathrm{Sb}-\mathrm{Bi}$ проводили на установке визуального термического анализа in situ по методике, описанной в работе [7].

Исследование упругих напряжений в эпитаксиальных слоях AlInSbBi и AlGaInSbBi проводили на основе стационарного поляризационно-оптического метода. Использовался инфракрасный микроскоп МИК-11 с электронно-оптическим преобразователем ЭОП-4 в поляризованном свете.

\section{4. Результаты и их обсуждение}

Влияние упругих деформаций кристаллической решетки на смещение фазовых переходов висмутсодер- 

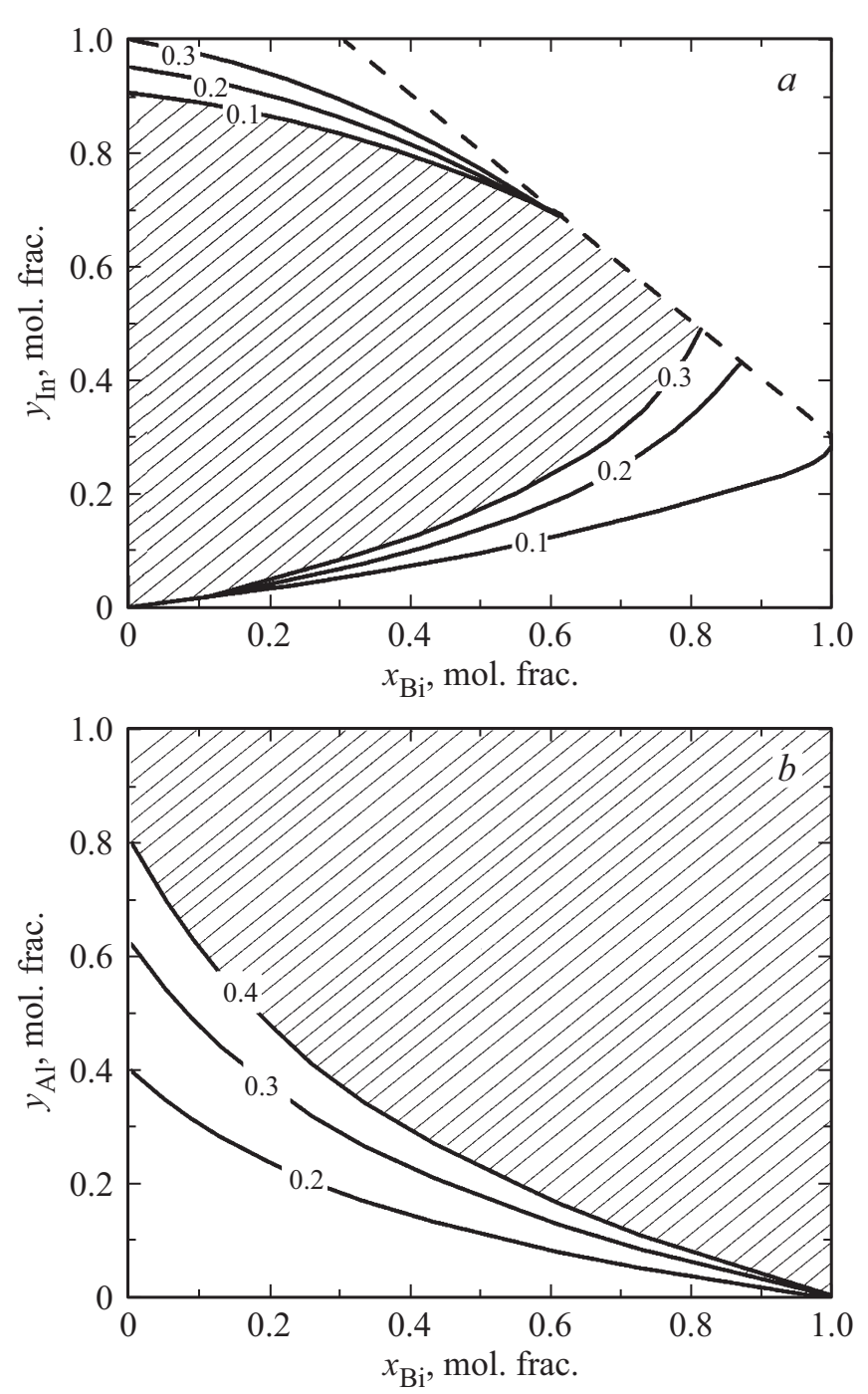

Рис. 6. Области спинодального распада гетероструктур: $a-$ $\mathrm{Al}_{y} \mathrm{In}_{1-y} \mathrm{Sb}_{1-x} \mathrm{Bi}_{x}$, значения концентрации $\mathrm{Al}$ указаны на кривых; $b-\mathrm{Al}_{y} \mathrm{Ga}_{z} \operatorname{In}_{1-y-z} \mathrm{Sb}_{1-x} \mathrm{Bi}_{x}$, значения концентрации $\mathrm{Ga}$ указаны на кривых.

жащих ТР показано на рис. 1. Кривые когерентного солидуса для $\mathrm{TP} \mathrm{Al}-\mathrm{In}-\mathrm{Sb}-\mathrm{Bi}$ близки к экспериментальным значениям, тогда как изотерма, рассчитанная по уравнениям РФД, соответствует эксперименту только в области малых значений $x_{\mathrm{In}}^{l}$. Поэтому на рис. 1 приведены изотермы солидуса раствора $\mathrm{Al}_{x} \mathrm{Ga}_{y} \mathrm{In}_{1-x-y} \mathrm{Sb}_{1-z} \mathrm{Bi}_{z}$, рассчитанные по КДС.

Получение висмутсодержащих твердых растворов $\mathrm{AlGaInSbBi}(\mathrm{InSb})$ и $\mathrm{AlInSbBi}(\mathrm{InSb})$ осложняется термодинамическими ограничениями, обусловленными наличием областей неустойчивости [6]. По описанной нами ранее методике [7] был проведен расчет областей устойчивости к распаду твердых растворов $\mathrm{AlGaInBi}(\mathrm{InSb})$ и $\mathrm{AlGaInSbBi}(\mathrm{InSb})$. Результаты расчетов представлены на рис. 6. Анализ областей термодинамической неустойчивости позволяет сделать выводы, что в область несмешиваемости при температурах эпитаксии попадает широкий диапазон составов твердых растворов, изопериодных подложкам бинарных соединений. Висмут существенно меняет область спинодального распада твердых растворов (заштрихованные области рис. $6, a$ ). Так, для

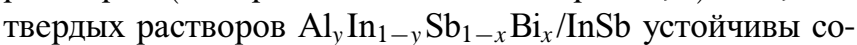
ставы с $0.7 \leq y_{\text {In }} \leq 1.0$ mol. frac., $0 \leq y_{\text {In }} \leq 0.5$ mol. frac. при $0 \leq x_{\mathrm{Bi}} \leq 1.0 \mathrm{~mol}$. frac. Однако упругие напряжения, возникающие на гетерогранице из-за рассогласования периодов решеток подложки и слоя, могут препятствовать спинодальному распаду. Висмут образует твердый раствор замещения в подрешетке сурьмы, деформируя решетку по типу растяжения [6]. Аналогичный эффект наблюдается при росте числа антиструктурных дефектов $\mathrm{Bi}_{\text {In }}$. Внедрение атомов $\mathrm{Bi}$ в междоузлия приводит к изгибу гетероструктуры в сторону кристаллизующегося слоя. Поэтому с ростом концентрации висмута в твердом растворе увеличивается напряженное состояние гетероструктуры и расширяется область термодинамической устойчивости (рис. 6). При введении в твердый раствор галлия в гетероструктуре $\mathrm{AlGaInSbBi/InSb} \mathrm{кро-}$ ме антиструктурных дефектов $\mathrm{Bi}_{\text {In }}$ появляются дополнительные дефекты $\mathrm{Bi}_{\mathrm{Ga}}$ и деформации решетки слоя, что в свою очередь приводит к увеличению внутренних напряжений гетероструктуры AlGaInSbBi/InSb, a, следовательно, к расширению области существования твердого раствора $\mathrm{AlGaInSbBi}$.

Результаты исследования упругих напряжений $\sigma$ от концентрации висмута в твердых растворах $\mathrm{AlInSbBi}(\mathrm{InSb})$ (рис. 7, кривые $1-3$ ) и $\mathrm{AlGaInSbBi}(\mathrm{InSb})$ (рис. 7, кривые 4-6) показали, что с увеличением концентрации висмута величина упругих напряжений

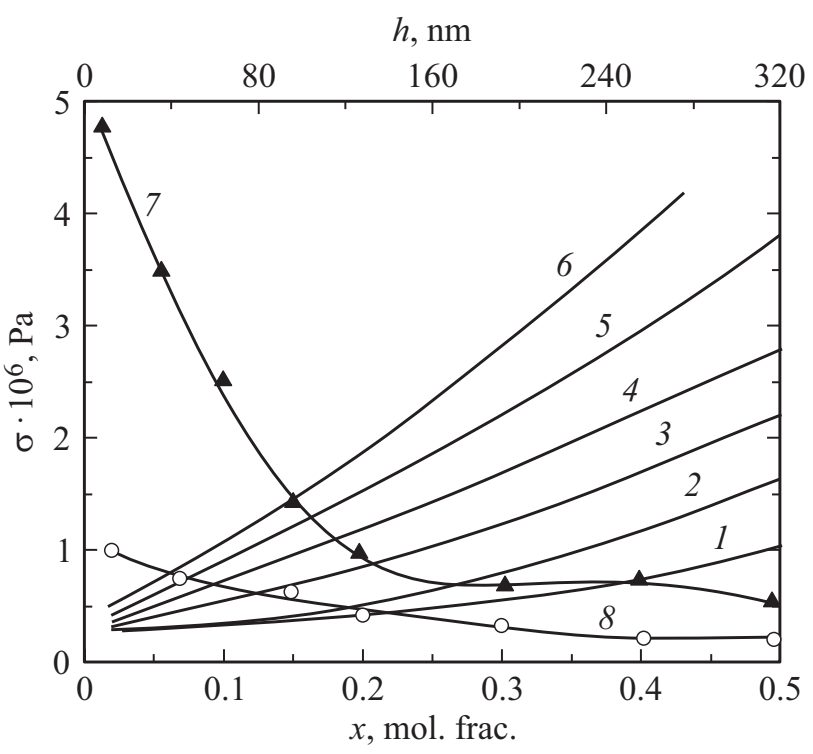

Рис. 7. Распределение упругих напряжений от концентрации Вi в гетероструктурах: $1-3-\mathrm{AlInSbBi} / \mathrm{InSb}$ : 4-6 - AlInGaSbBi/InSb; кривые: $1,4-G=10 \mathrm{~K} / \mathrm{cm}$, $2,5-G=20 \mathrm{~K} / \mathrm{cm}, 3,6-G=30 \mathrm{~K} / \mathrm{cm} ; 7-$ для варизонных гетероструктур AlInGaSbBi/InSb; 8 - для гетероструктур AlInGaSbBi/InSb, выращенных с подпиткой. 
возрастает, особенно при $x_{\mathrm{Bi}}>0.25 \mathrm{~mol}$. frac. Добавление $\mathrm{Ga}$ делает твердый раствор AlInSbBi более напряженным (рис. 7, кривые 4-6). Результаты исследований $\sigma$ по толщине варизонных пленок $\mathrm{AlGaInSbBi}(\mathrm{InSb})$ (рис. 7, кривая 7) показали значительное ухудшение структурного совершенства на границе гетероструктуры. Это связано с тем, что в приграничной области состав значительно изменяется, что ведет к увеличению рассогласования периодов решеток слоя и подложки, а также к возникновению дефектов и напряжений. Такой эффект отсутствует в гетероструктурах $\mathrm{AlGaInSbBi/InSb}$ постоянного состава (рис. 7, кривая 8).

\section{5. Заключение}

Комплексным анализом фазовых переходов в упруго-напряженных гетеросистемах $\mathrm{Al}-\mathrm{Ga}-\mathrm{In}-\mathrm{Sb}-\mathrm{Bi}$ и подсистемах $\mathrm{In}-\mathrm{Sb}-\mathrm{Bi}, \mathrm{Al}-\mathrm{In}-\mathrm{Sb}-\mathrm{Bi}$ установлена область существования твердых растворов AlGaInSbBi без спинодального распада. Удовлетворительное согласование с экспериментальными данными позволяет говорить об адекватности модели расчета фазовых переходов в исследованной гетеросистеме.

Введение висмута в твердый раствор AlGaInSbBi pacширяет область его существования за счет увеличения относительного решеточного рассогласования слоя и подожки и, следовательно, уменьшает пределы составов изопериодных гетероструктур $\mathrm{AlGaInSbBi} / \mathrm{InSb}$.

Экспериментальные исследования структурного совершенства гетероструктур AlInSbBi/InSb и AlGaInSbBi/ $\mathrm{InSb}$ показали, что величина упругих напряжений $\sigma$ увеличивалась с ростом содержания висмута в твердом растворе. В варизонных слоях AlGaInSbBi дополнительным источником упругих напряжений могут служить значительные градиенты состава по толщине слоев.

\section{Финансирование работы}

Исследования концентрационных зависимостей и спинодальных распадов выполнены при финансовой поддержке Российского научного фонда (грант № 19-79-10024), исследования распределения упругих напряжений выполнены за счет средств РФФИ (грант № 20-08-00108 A).

\section{Конфликт интересов}

Авторы заявляют, что у них нет конфликта интересов.

\section{Список литературы}

[1] L. Wang, L. Zhang, L. Yue, D. Liang, X. Chen, Y. Li, P. Lu, J. Shao, S. Wang. J. Cryst. 7, 63, (2017).

[2] Д.Л. Алфимова, Л.С. Лунин, М.Л. Лунина, А.С. Пащенко, С.Н. Чеботарев. Неорган. материалы 53, 1, 33, (2017).

[3] A.S. Pashchenko, L.S. Lunin, E.M. Danilina, S.N. Chebotarev. Beilstein J. Nanotechnol. 2018, 9. 2794 (2018).
[4] S. Malyshev, A. Chizh. IEEE J. Select. Top. Quantum Electron. 10, 4, 679, (2004).

[5] S. Malyshev, A. Chizh. IEEE J. Lightwave Tech. 25, 11, 3236, (2007).

[6] В.Н. Лозовский, Л.С. Лунин, А.В. Благин. Градиентная жидкофазная кристаллизация многокомпонентных материалов. СКНЦ ВШ, Ростов н/Д (2003). 374 с.

[7] В.В. Кузнецов, Л.С. Лунин, В.И. Ратушный. Гетероструктуры на основе четверных и пятерных твердых растворов соединений $\mathrm{A}^{\mathrm{III}} \mathrm{B}^{\mathrm{V}}$. СКНЦ ВШ, Ростов н/Д. (2003). 376 с.

Редактор Д.В. Жуманов 